\title{
Impact of donor kidney weight to recipient body weight ratio on long-term graft outcomes in live donor kidney transplantation
}

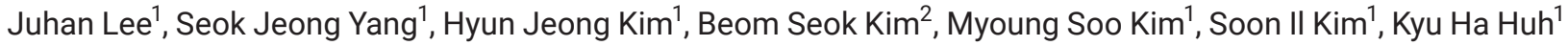 \\ ${ }^{1}$ Department of Surgery-Transplantation, Yonsei University College of Medicine, Seoul, Korea \\ ${ }^{2}$ Department of Internal Medicine-Nephrology, Yonsei University College of Medicine, Seoul, Korea
}

Background: Kidney weight has been suggested as a surrogate marker for nephron numbers and renal function. Small donor kidney sizes relative to recipient body size is an important contributor to short-term graft renal function, but the impact of size mismatching on long-term graft outcomes remains unknown. This study is aimed to evaluate the impact of the donor kidney weight to recipient body weight ratio (KW/BW) on long-term graft survival in live donor kidney transplantation.

Methods: We performed a longitudinal cohort study in 1,397 patients who underwent live donor kidney transplantation between 2000 and 2016 at a single center. Following cold perfusion and back table surgery, the kidney was weighted on the same electronic weighting scale by the surgeon. Patients were grouped into four groups according to KW/BW quartiles.

Results: During a median follow-up of 127 months, 245 graft loss occurred (172 graft failures and 73 patient deaths). The 10-year death-censored graft survival rates were $86.9 \%$ in the lowest quartile, $90.4 \%$ in the second quartile, $90.5 \%$ in the third quartile, and $92.4 \%$ in the highest quartile $(P=0.002)$. Multivariable analysis revealed that the lowest KW/BW (hazard ratio [HR], 2.16; 95\% confidence interval $[\mathrm{Cl}], 1.33-3.48 ; \mathrm{P}=0.002)$ and second lowest $\mathrm{KW} / \mathrm{BW}(\mathrm{HR}, 1.69 ; 95 \% \mathrm{Cl}, 1.06-2.70 ; \mathrm{P}=0.029)$ groups were significantly associated with death-censored graft failure compared with the highest KW/BW group. Patients with lower KW/BW exhibited consistently lower estimated glomerular filtration rates than those with higher KW/BW.

Conclusions: Low KW/BW is significantly associated with long-term graft outcome in live donor kidney transplantation.

Corresponding author: Juhan Lee

E-mail: laplaine@yuhs.ac

(c) The Korean Society for Transplantation

This is an Open Access article distributed under the terms of the Creative Commons Attribution Non-Commercial License (http://creativecommons.org/licenses/by-nc/4.0/) which permits unrestricted non-commercial use, distribution, and reproduction in any medium, provided the original work is properly cited. 